\title{
Control de Broca de Café (Hypothememus hampei) por medio del microorganismo benéfico Beauveria bassiana en la zona de reserva La Botija, San Marcos de Colón
}

\author{
Claudia Patricia Sierra Molina*, Liliam Carina Marroquín **
}

\section{RESUMEN}

El café es uno de los principales productos agrícolas de exportación de Honduras; la broca del fruto del café es una plaga de insectos conocida científicamente como Hypothememus hampei, y en su forma de adulto, el insecto es un pequeño escarabajo de coloración oscura con un cuerpo cilíndrico, robusto, recurvado en la región posterior. En Honduras esta plaga se cita por primera vez en 1977, afectando un área aislada aproximadamente de 300 manzanas, implicando importantes pérdidas en los rendimientos por cosecha. Para lo cual ha desarrollado un programa de control de manejo integrado, fundamentado en la biología de la broca y su relación con el desarrollo del fruto, que se combina con control cultural y un control biológico.

El control biológico usado es el hongo Beauveria Bassiana que es un parásito de las brocas que después de 3 a 6 días, en condiciones de humedad saturada, causa infecciones y posteriormente su muerte, confirmando los resultados positivos durante el ensayo en los lotes seleccionados.

\section{Palabras clave: Beauveria Bassiana, café, Broca (Hypothememus hampei)}

\section{ABSTRACT}

Coffee is one of the main agricultural exports of Honduras. The coffee berry borer is a plague of insects known scientifically as Hypothememus hampei. In its adult aspect this insect is a small dark-colored beetle with a cylindrical body form, robust and curved in the posterior region. In Honduras this plague was reported for the first time in 1977 when it affected an isolated area of 300 blocks, involving large losses in crop

Universidad Nacional Autónoma de Honduras. Centro Universitario Regional del Litoral Pacífico. Departamento de Agroindustria. Carrera de Ingeniería Agroindustrial.

" Universidad Nacional Autónoma de Honduras. Centro Universitario Regional del Litoral Pacífico. Departamento de Agroindustria. Carrera de Ingeniería Agroindustrial. Asesora. carimarroquin@yahoo.es 
yields. This developed an integrated management control, based on the biology of the drill and its relationship with the fruit development, which has been combined with a cultural and a biological control.

The biological agent used is the fungus Beauveria bassiana, which is a parasite of the drill that causes infection and death after 3 to 6 days in humid saturated conditions, confirming positive results in the testing of the selected batches

Key Words: Beauveria bassiana, coffee, Drill (Hypothememus hampei) 


\section{INTRODUCCIÓN}

Hypothememus hampei (Coleóptera: Curculionidae) es la plaga más perniciosa del café a nivel mundial (Bustillo et al., 1998). Damón indicó que a pesar de los avances en la investigación con respecto a la broca, el control de la plaga dependía ampliamente de la aplicación del insecticida órganoclorado endosulfán, con los que se han obtenido resultados variables e impredecibles (Damón. 2000) se señaló la necesidad de la búsqueda de sustitutos de los agroquímicos, y en este sentido se ha orientado el interés hacia los insecticidas de la tercera generación y dentro de éstos al grupo de mediadores biológicos, que son productos naturales que intervienen en las relaciones hospedero-parásitos. Entre las medidas de control más comunes contra la broca están la utilización de entomopatógeno (Monzón, 2001), siendo Beauveria bassiana el más conocido y aplicado con éxito en programas de manejo integrado de plagas. (Cárdenas 2000).

Se destacó que dentro de los controles de $\underline{H}$. hampei, el control cultural es el componente más importante en el manejo integrado de esta plaga. (Benavides et al. 2002). Al mismo tiempo que se realizaron evaluaciones para verificar la efectividad que ejerce el hongo Beauveria bassiana sobre dicha plaga con el fin de concientizar y facilitar acerca de los resultados obtenidos a los pequeños productores de café, para que empleen este mecanismo de control biológico y evitar el uso de plaguicidas, insecticidas u otro químico que ocasiona daños ambientales y a la salud humana. (Comunicación personal, Msc. Venessa. 2010), y así, para brindar un producto seguro para los consumidores.

Para comprobar la hipótesis de este estudio se optó por un enfoque cuantitativo en el que se hizo uso de la medición numérica y la recolección de datos estadísticos, utilizando un diseño factorial y teniendo un alcance correlacional por que permitió conocer el comportamiento de las variables tanto independientes: hongo Beauveria bassiana y condiciones físicas del lugar y la variable dependientes: plantas de café brocadas, del estudio de la broca en la finca seleccionada, teniendo como objetivo cómo evaluar el control de la broca del café por medio del hongo benéfico Beauveria bassiana en zona de reserva, montaña La Botija, San Marcos de Colón y determinar la efectividad del hongo Beauveria bassiana sobre esta plaga.

\section{MÉTODO}

\section{Diseño}

Es un estudio experimental, analítico descriptivo, observacional y transversa,I utilizando dos parcelas de cultivo con 100 plantas por cada parcela. 


\section{Población}

El estudio se realizó en dos parcelas de café con 100 plantas para cada una, haciendo un total de 200 plantas evaluadas, una parcela para control y otra en la que se aplicó el control biológico.

\section{Entorno}

Este estudio se realizó en la finca La Reforma, Aldea Mesas de Cacamuya, situada en San Juan de Duyusupo, zona de reserva La Botija, San Marcos de Colón, en un área de 1.5 manzanas, terreno plano con altura de $1300 \mathrm{msnm}$, con precipitación anual de 1200 a $1400 \mathrm{~mm}$, con temperaturas anuales de $25^{\circ} \mathrm{C}$, sombra diversa, en las variedades de café Caturra y Típico con edades de 10 años. El estudio se llevó a cabo durante el periodo de septiembre a diciembre.

\section{Control Cultural}

Comprende la aplicación oportuna de las tecnologías apropiadas de manejo del cultivo, especialmente regulación de sombra, poda, deshierba, recolección de frutos brocados.

\section{- Regulación de sombra:}

La broca ataca intensamente a los frutos y se reproduce rápidamente en los cafetales con excesiva sombra, poca ventilación interna y deficiente cuidado del cultivo. Esta labor consiste en podar los árboles, deshijar y deshojar las plantas de banano, guineo u otras especies que proporcionan sombra al cafetal.

\section{- Poday deshierba:}

Es la eliminación de chupones, ramas improductivas y paloteadas, así como de las hojas severamente enfermas. Mediante las deshierbas oportunas se evita la competencia de las malezas con el cafeto por espacio, agua, luz y nutrientes.

\section{- Recolección de los frutos brocados:}

Los frutos brocados deben ser cuidadosamente recolectados de la planta así como los caídos al suelo. Los frutos plagados, luego de ser recolectados, deben ser quemados o sumergidos en agua en altas temperaturas. La ejecución de esta labor cultural, proporciona una adecuada luminosidad y aeración al cafetal, creando condiciones favorables para el ataque de la broca y otras plagas del café. (Comunicación personal, técnico Pedro. 2010) 


\section{Preparación y aplicación de la suspensión de Beauveria bassiana}

Se vierte el producto sobre un colador con el Beauveria bassiana en un balde plástico o metálico, se remueve fuertemente para separar las esporas. Posteriormente, el agua con las esporas se pasa por un colador fino y se agrega a un barril de 200 litros de agua. Luego, la suspensión se aplica con ayuda de una bomba de mochila directamente sobre los frutos. Debe asegurarse que el equipo no haya sido utilizando para aplicación de fungicidas. (Montilla R. Camacho B. Quintero A. Cardozo G.)

\section{Variables evaluadas}

\section{Porcentaje de broca en el fruto de café dañado durante la cosecha}

Para determinar el porcentaje de infestación por la broca en los cafetales se seleccionaron 100 árboles de café, y se recorrieron en forma aleatoria, en cada árbol se seleccionó una rama y se contaron todos los frutos, marcando los árboles según la muestra, luego se contaron los frutos brocados y se determinó el porcentaje de infestación de broca utilizando la siguiente operación:

$$
\begin{gathered}
\%=\text { NÚMERO DE FRUTOS } \\
\text { BROCADOS X } 100 \\
\text { NÚMERO TOTAL DE FRUTOS }
\end{gathered}
$$

\section{Porcentaje de efectividad del control natural por Beauveria bassiana}

Después de 4 días se aplicó el hongo en el muestreo de los frutos brocados con la Beauveria bassiana, en forma aleatorea utilizando esta fórmula, para obtener el porcentaje de efectividad.

\section{\%=NÚMERO FRUTOS BROCADOS CON BEAUVERIA BASSIANA X100 NÚMERO DE FRUTOS BROCADOS}

\section{RESULTADOS YDISCUSIONES}

\section{Porcentaje de infestación antes y después del tratamiento}

En ambos lotes, tanto el del tratamiento como en el del control, se obtuvo en promedio un $32 \%$ de infestación de Broca del Café por cada lote, al aplicar el control biológico se encontró que el lote, donde se había aplicado el hongo, no se 
encontraron frutos brocados por lo que podemos decir que el control biológico fue efectivo para el control de broca en el cultivo de café y en el lote control donde solo se realizaron labores culturales presentó un $5 \%$ de broca después de los siete días que se esperó para determinar la efectividad del hongo; eso implica que si no usamos control biológico, perderemos un $5 \%$ de la producción de cada lote lo que implica pérdida para el productor tanto de producto como de calidad, mientras que si usamos control biológico, es evidente que revertimos este $5 \%$ por lo que el café obtenido será de mejor calidad.

Ambos procedimientos, tanto el de labores culturales como el control biológico, son efectivos en el tratamiento de la broca, pero si usamos ambos es un cinco porciento más efectivo que solo el uso de labores culturales, lo que garantiza la producción del caficultor así como la calidad del producto ya que se producirá café sin daños y no habrá más pérdidas causadas por la infestación de la broca.

\section{CONCLUSIÓN}

Una combinación de labores culturales y control biológico con Beauveria bassiana son un efectivo mecanismo en el control de Broca del Café, su uso evitará pérdidas en la producción y mejorará la calidad de los frutos obtenidos.

\section{BIBLIOGRAFÍA}

- Agronomía, Recursos Forestales Montes. "Control Biológico de la Broca del café" .http://html.rincondelvago.com/control.biologico-de-la-broca....-34k. 17 julio 2010.

- Aguirre Saltarrea F. "Plagas del cafeto y problemas climáticos" www.buscocafe.com 30 de septiembre 99.

- Chege K. "Plaga del café, lista para aprovechar giro climático".http://agronomord.blogspot.com/2009/09/cambio-climaticofavo. 15 de julio 2010.

- Dr. Klein Kouch C. "La Broca del café." www.infoagro.net/shared/docs/a3/ Broca-del-café.pdf. 28 junio 2010.

- Fernández S. y Cordero J. "Biología de la Broca del café en condiciones de laboratorio".http://seder.col.god.mx/direcciones/dsanidad/semanasanidad. Accesado 17 de julio 2010.

- Forero Lleras Laura. "técnicas para identificar la zona flaqueante del TRANSPOSON del HUPFER en Beauveria bassiana". www.javeriana.edu.co/biblios/tesis/ciencias. 
- Fundación Salvadoreña para investigadores del café. "Manejo Integrado de la Broca del fruto". www.pracafe.com.sv/.../ManejorlntegradoBroca:htm. 4 julio 2010.

- Zenner I, Posadas F, Vélez M. Universidades de ciencias aplicadas y ambientales. "Hormigas: enemigas naturales de la broca del café". http://especiales. universia.net.co/libro-abierto/agronomia-veterinaria-yafines/hormigas-enemigos-naturales-de-la-broca-del-cafe.html. Accesado 7 julio 2010.

Formulación sólida a base de Bacillus Thuringiensis para el 